\title{
Review of: "Prevalence of common carbapenemase genes and multidrug resistance among uropathogenic Escherichia coli phylogroup B2 isolates from outpatients in Wasit Province/ Iraq"
}

\author{
Angel Manjarrez-Hernandez ${ }^{1}$ \\ 1 Universidad Nacional Autónoma de México
}

Potential competing interests: The author(s) declared that no potential competing interests exist.

The carbapenemresistant strains have become a serious public health issue in the worldwide and are usually resistant to almost all antibiotics. This article shows high occurrence of carbapenemase genes, among phylogroup B2 uropathogenic E. coli isolates from patients with urinary tract infections in Iraq. The most frequently detected carbapenemase genes were blaOXA and blapER type, in other parts of the world, in addition to these genes, different carbapenemase genes have been detected among $E$. coli isolates. Interesting, in this study all the strains were susceptible to carbapenems antibiotics but were multidrug resistance, as if these bacteria were adapting in their environment to become carbapenem-resistant phenotype. This possibility is not far from reality considering that plasmid-mediated carbapenemresistance is widely perceived as attributing to the dissemination of the carbapenem-resistant genes and the emergence of carbapenem-resistant strains. The carbapenem-resistant genes could co-exist with $\beta$ lactamase and other resistant genes on plasmid, which brought a new challenge to the treatment of infections caused by carbapenem-resistant strains. 